\title{
In situ nuclear morphology measurements using light scattering as biomarkers of neoplastic change in animal models of carcinogenesis
}

\author{
Adam Wax* and John W. Pyhtila \\ Department of Biomedical Engineering, Fitzpatrick Institute for Photonics, Duke University, Durham, NC, USA
}

\begin{abstract}
Light scattering spectroscopy measurements can be used to determine the structure of tissue samples. Through refined data acquisition and signal processing techniques, quantitative nuclear morphology measurements may be obtained from light scattering data. These data have been used primarily as a biomarker of neoplastic change in a wide range of settings. Here, we review the application of light scattering to assessing the health status of tissues drawn from animal models of carcinogenesis, in particular, the rat esophagus and the golden Syrian hamster trachea carcinogenesis models. In addition, we present results from ex vivo human tissues to demonstrate the relevance of the use of animal models which are excellent surrogates for several human cancers. These models provide the opportunity to develop biomarkers and test chemopreventive and therapy strategies before application in humans.
\end{abstract}

Keywords: Light scattering measurements, neoplastic change, carcinogenesis, chemoprevention

\section{Introduction}

Animal models are essential to cancer research for their ability to improve understanding of the carcinogenesis process as well as for their capacity to aid in the development of chemopreventive and chemotherapeutic agents which retard or regress malignant change. A crucial need in this field is the identification of biomarkers which monitor the progression of tissue transformation in animal carcinogenesis models. As an example, most evaluations of chemopreventive agents are based on tumor incidence, multiplicity and size data obtained by gross necropsy of tissues from animal carcinogenesis models $[1,5,12,15,25]$. This approach has the drawback that an endpoint consisting of a visible tumor must be reached to determine efficacy. Other-

${ }^{*}$ Corresponding author. Tel. +1 919 6605143; E-mail: a.wax@ duke.edu. wise, histopathological determination of the frequency of pre-neoplastic lesions must be used, requiring the visual evaluation of a large number of slides by a trained expert. Further, this approach requires sacrifice of the animal to enable the analysis, which precludes subsequent monitoring of further neoplastic change. As alternatives to gross necropsy, other biomarkers of cancer development are often sought, including cytological washings [12], and analysis of DNA and protein adducts in blood components [8,14,24]. These techniques avoid the need for animal sacrifice but are not as developed or widely applicable.

Promising new avenues for monitoring cancer progression based on optical imaging techniques are under development using small animal models [3,4,13]. Such techniques employ fluorescent tags which exhibit preferential uptake by tumors. However, the interaction between the fluorescent tags and potential chemopreventive agents is unknown and may limit the applicability of these imaging techniques for tracking effi- 
cacy. The use of intrinsic contrast mechanisms, such as those used in light scattering techniques, can circumvent these difficulties and provide a useful alternative for evaluating the efficacy of chemopreventive agents.

Recently, light scattering techniques were shown to be capable of detecting neoplastic transformation in animal models $[23,28,31]$. These techniques utilize the interaction between light and cellular structures to obtain quantitative information on nuclear morphology [28] or tissue micro/nano-architecture [23] in epithelial layers without the need for biopsy and histopathologic evaluation. These non-invasive, nonperturbative techniques provide a unique opportunity to study the biology of neoplastic progression in living tissue, free from artifacts associated with preparation of histopathological slides for diagnostic evaluation, and at times before the tumors become visible.

Presented here is a review of the development of a novel light scattering technique, angle-resolved low coherence interferometry $\left(\mathrm{a} / \mathrm{LCI}^{1}\right)$, and its application to monitoring neoplastic progression through measurements of nuclear morphology [28,31]. The a/LCI technique obtains nuclear structural information by examining the angular distribution of backscattered light. With a/LCI, depth resolution is achieved by the use of a short coherence length light source in an interferometry scheme. This method of coherence gating is also employed to obtain depth resolution in optical coherence tomography (OCT), a technique used to construct tomographic images of living tissues $[9,11]$. However, in contrast to OCT, a/LCI has the added feature of resolving the angular distribution of scattered light, permitting structural measurements which exceed the resolution obtained with OCT. In a/LCI measurements, just as in OCT, the depth resolution serves as a powerful means of rejecting multiply scattered light, enabling selective measurements of light which has interacted only with a tissue region of interest. This capability has been exploited to reject the light scattered by the suprabasal layers and examine exclusively the morphology of basal cell nuclei, which is where precancerous growth begins.

The remainder of this review is organized as follows. First, an overview of the a/LCI technique is presented, including the optical systems, signal processing and

\footnotetext{
${ }^{1}$ The abbreviations used are: a/LCI, angle-resolved low coherence interferometry; NMBA, N-nitrosomethylbenzylamine; REC rat esophageal carcinogenesis DFMO, difluoromethylornithine; DMSO, dimethyl sulfoxide; LGD, low-grade dysplasia; HGD, highgrade dysplasia.
}

data analysis methods. This is followed by a review of the application of the technique to tracking neoplastic transformation in animal models of carcinogenesis, specifically the rat esophagus and hamster trachea models, through nuclear morphology measurements. These previous studies using a/LCI to study animal models have focused on the nuclear diameter as the primary biomarker for assessing neoplastic change. However, in the current review, we will emphasize the utility of a second nuclear morphology descriptor, the refractive index which was not previously examined in detail in previous studies. The refractive index of the cell nucleus relative to the cytoplasm is a parameter that can be extracted from the light scattering data which can be related to the density of the cell nucleus. Here we identify that the relative refractive index of the nucleus is a valuable biomarker of neoplastic change through analysis of the data from these previous studies. This is followed by a review of the application of a/LCI to examining ex vivo human tissues to demonstrate the relevance of the animal models. Finally, we will conclude with a summary and a look at future directions of light scattering research for detecting neoplastic change.

\section{Angle-resolved low coherence interferometry (a/LCI) - Experimental schemes}

The a/LCI technique has been developed as an elastic light scattering technique for detecting nuclear morphology in intact, unstained tissues. A central feature of elastic light scattering methods is the need to isolate the light which has only interacted a single time with the sample. In a/LCI, this is achieved using coherence gating, implemented with an interferometry scheme. Since its inception, there have been three designs of the a/LCI method. The first a/LCI prototype was designed to demonstrate the proof of principle behind the method and did not achieve fast scan times, but required 30 minutes to examine tissue regions of $1 \mathrm{~mm}^{2}$. The second generation a/LCI system improved upon this by changing the system design and realized scan times of approximately 5 minutes for a similar tissue region. The most recent implementation of the method uses an endoscopic fiber bundle and a frequency domain data acquisition scheme to enable data acquisition times of less than 50 milliseconds while demonstrating clinical feasibility for endoscopic applications. We now briefly review these three implementations.

The original a/LCI prototype (Fig. 1(a)) is based on a modified Michelson interferometer geometry which 
used a superluminescent diode $\left(\lambda_{\mathrm{o}}=845 \mathrm{~nm}\right.$, FWHM bandwidth $22 \mathrm{~nm}$ ) as a light source [27,29]. Light from the SLD is divided by a beamsplitter (BS) into a reference beam and an input beam to the sample. The reference beam is reflected by a mirror (M) and recombined at BS with light reflected by the sample. The mixed fields generate an interference pattern when that the two optical path lengths are matched to within the coherence length of the source $\left(l_{c}=2 \ln 2 / \pi \times\right.$ $\left.\lambda^{2} / \Delta \lambda=14.3 \mu \mathrm{m}\right)$. This mechanism of coherence gating allows depth resolved reflection profiles to be generated by changing the path length of the reference field. The interference signal is isolated by heterodyne detection. A Doppler shift of $9.5 \mathrm{kHz}$ is imparted to the reference beam by translating the reference mirror (M) at a constant velocity $(4 \mathrm{~mm} / \mathrm{s})$. Upon mixing of the reference and signal fields, a heterodyne signal is generated. The heterodyne beat signal is isolated by calculating the power spectrum of the digitized photocurrent (sampled at $333 \mathrm{kHz}$ ) and bandpassing the signal around the heterodyne frequency.

The measurement of the angular distribution of scattering is achieved by the unique imaging system of the original prototype a/LCI system, comprised of four achromatic imaging lenses (L1-L4, focal lengths $\left.f_{1}-f_{4}\right)$. These lenses are arranged to form multiple $4 f$ imaging systems, which image both the phase and amplitude of the light field. The reference field is made to cross the detector plane at a variable angle by scanning lens L2 $\left(f_{2}=4.5 \mathrm{~cm}\right)$ a distance $\Delta$ y perpendicular to the beam path. It can be shown using Fourier optics that this translation causes the reference field to be reproduced in the plane of the detector (D) with its angle of propagation changed by $\theta_{\mathrm{T}}=2 \Delta y / f_{2}$ but its position unchanged [27].

The second generation a/LCI system (Fig. 1(b)) is based on a Mach-Zehnder interferometer which used a mode-locked Ti:Sapphire laser $\left(\lambda_{\mathrm{o}}=830 \mathrm{~nm}\right.$, FWHM bandwidth $10 \mathrm{~nm}$ ) as a light source. The advantage of this new geometry is that it enables the use of acoustooptic modulators (AOM's) to create the offset in frequency that will generate a heterodyne beat signal when the two fields are mixed. The light from the source is split into signal and reference fields by a beamsplitter and each given a frequency shift of 110 or $120 \mathrm{MHz}$. This results in a heterodyne beat at $10 \mathrm{MHz}$ when the fields are mixed at beamsplitter BS2. By exploiting the short coherence length $(30 \mu \mathrm{m})$ of the source, the detected signal field is resolved by its optical path length. Information about the signal field is obtained at various depths within the sample by translating retroreflec-

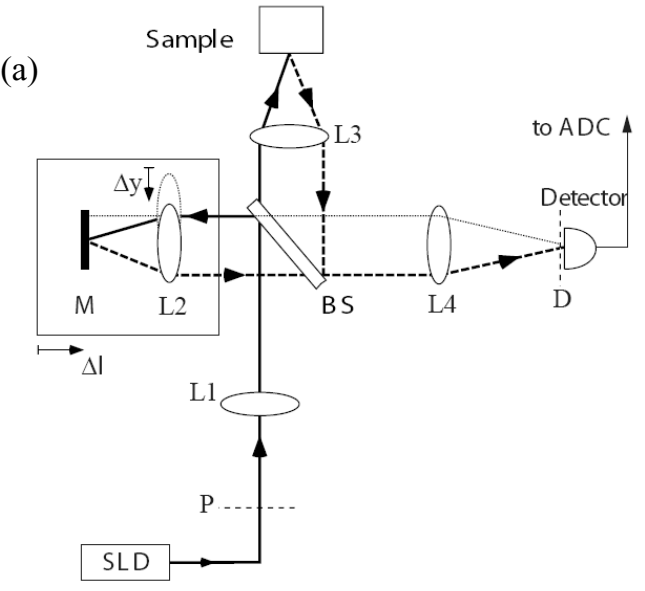

(b)

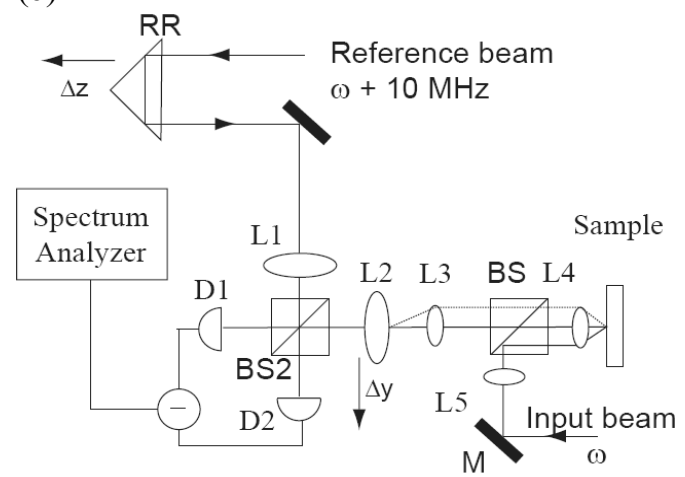

Fig. 1. Schematic of a/LCI systems: (a) original prototype system, (b) second generation system. In both systems, translation of lens L2 enables the angle of the detected scattering signal to be varied. (Taken from Ref [31]).

tor $(\mathrm{RR})$ to vary the path length of the reference field by $2 \Delta z$. However, unlike the previous system, the translation of the retroreflector does not appreciably influence the frequency of the heterodyne signal. Since the signal is now independent of variations in the scan speed of the retroreflector, the frequency of the heterodyne beat is more stable, resulting in a higher fidelity measurement. In addition, the system uses a balanced detector (D1 and D2, New Focus Model 1807) and demodulates the photocurrent using a spectrum analyzer (HP 8594E) resulting in better signal-to-noise ratio and faster acquisition times than the previous a/LCI prototype while offering the same precision and accuracy for obtaining structural information [22].

The second generation system measures the angular distribution of the scattered field by scanning lens L2 a distance $\Delta y$ perpendicular to the beam path. It has 


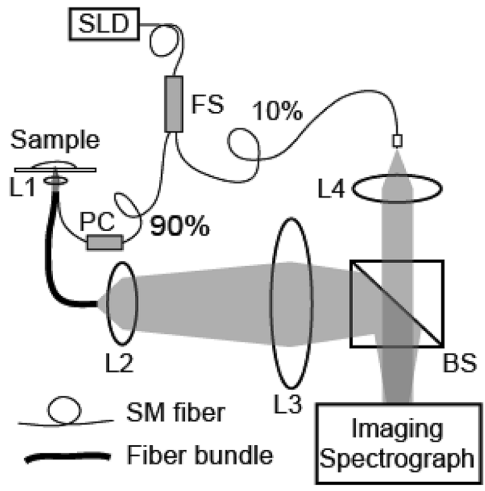

(a)

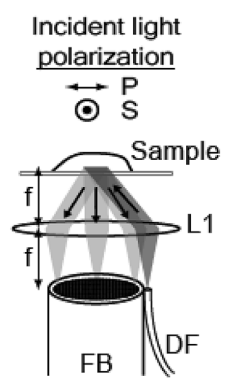

(b)
Fig. 2. (a) Schematic of frequency domain a/LCI system. Shaded areas indicate light paths. (b) Sample illumination and scattered light collection with the distal end of the probe. (Taken from Ref [19]).

been shown using Fourier optics that this translation will permit selective detection of the light which arrives at the plane of L2 traveling at an angle $\theta \Delta y / f_{2}$ relative to the optical axis, where $f_{2}=10 \mathrm{~cm}$ is the focal length of L2 [26]. Lens L1 $\left(f_{1}=10 \mathrm{~cm}\right)$ is included to alter the wavefront of the reference field to compensate for the effects of L2. Lenses L3 and L4 form a $4 f$ system, imaging both the phase and amplitude of the scattered field onto the plane of lens L2. Lens L5 $\left(f_{5}=10 \mathrm{~cm}\right)$ is included so that L4 does not focus the input beam on the sample but instead re-collimates it into a pencil beam.

The most recent implementation of a/LCI is based on a fiber optic interferometer and employs frequency domain detection [21]. The use of fiber optics opens the door for endoscopic application of the a/LCI technique, while the use of frequency domain detection enables fast enough data acquisition times (50 milliseconds) to make the approach feasible as an in vivo diagnostic of cancer in humans. The frequency-domain a/LCI system (fa/LCI, Fig. 2(a)) is based on a modified MachZehnder interferometer. Unlike the previous a/LCI systems which relied on the generation of a heterodyne signal to detect the interference, the new system uses frequency domain data acquisition [20]. This approach has been shown to produce higher quality signals by collecting data from all depths simultaneously rather than scanning the region of interest depthwise in a serial fashion.

The endoscopic fa/LCI system uses light from a fiber-coupled superluminescent diode (SLD) source (Superlum, $\mathrm{P}_{\mathrm{o}}=5 \mathrm{~mW}, \lambda_{\mathrm{o}}=841.5 \mathrm{~nm}, \Delta \lambda=$ $49.5 \mathrm{~nm}$, coherence length $=6.3 \mu \mathrm{m}$ ) which is split into sample and reference arms by a $90 / 10$ fiber splitter
(AC Photonics). The polarization controller, $\mathrm{PC}$, in the sample arm is adjusted to produce linearly polarized light either parallel $(P)$ or perpendicular $(S)$ to the vector of angular scattering data collected, as illustrated in Fig. 2(b). Due to the birefringence in the fiber, it is necessary to precisely control the polarization of the incident light in order to maintain knowledge of the polarization state for data analysis [19].

The system enables detection of the angular distribution of scattered light by the configuration of the probe tip. The sample probe and the sample (Fig. 2(b)) are in conjugate Fourier planes of lens L1, resulting in a $0.5 \mathrm{~mm}$ diameter collimated beam incident on the sample at an oblique angle, and producing a means for collecting the angular distribution of the scattered field at the distal face of the multimode coherent fiber bundle (Schott North America, Inc., length $=840 \mathrm{~mm}$, pixel size $=8.2 \mu \mathrm{m}$, pixel count $=13.5 \mathrm{~K}$ ). As an illustration, the optical path of the incident light and light scattered at three selected scattering angles are shown in Fig. 2(b). The angular range of the system is experimentally determined to be 0.12 to $0.45 \mathrm{rad}$.

The fiber bundle is spatially coherent, resulting in a reproduction of the collected angular scattering distribution at the proximal face. Additionally, as all fibers in the bundle are path length matched to within the coherence length of the source, the optical path length traveled by scattered light at each angle is identical. It is experimentally determined that the higher order modes propagating in the multimode fibers are offset from the fundamental mode by at least $3.75 \mathrm{~mm}$ in optical path length. Additionally, the power in the higher order modes have a minimal affect on dynamic range, as the power of the scattered light collected by the fiber bundle is significantly less than the reference arm power. The angular distribution exiting the proximal end of the fiber bundle is relayed by the $4 \mathrm{f}$ imaging system of L2 and L3 $\left(f_{2}=3.0 \mathrm{~cm}, f_{3}=20.0 \mathrm{~cm}\right)$ to the input slit of the imaging spectrograph (Acton Research, InSpectrum 150), where the angle of scattering, $\theta$, is linearly mapped to a vertical position on the input slit.

The optical path length of the reference arm is matched to that of the fundamental mode of the light in the sample arm. Light exiting the reference fiber is collimated by lens L4 $(\mathrm{f}=3.5 \mathrm{~cm}$, spot size $=8.4 \mathrm{~mm})$ and mixed with the angular scattering distribution at beamsplitter BS. The mixed fields are dispersed with a high resolution grating (1200 lines $/ \mathrm{mm})$ and detected using an integrated, cooled CCD $(1024 \times 252,24 \mu \mathrm{m}$ $\times 24 \mu$ m pixels, $0.1 \mathrm{~nm}$ resolution) covering a spectral range of $99 \mathrm{~nm}$, centered at $840 \mathrm{~nm}$. The detected sig- 
nal, a function of wavelength, $\lambda \mathrm{s}$ and $\theta$, can be related to the signal and reference fields $(E s, E r)$ as:

$$
\begin{aligned}
& I\left(\lambda_{m}, \theta_{n}\right)= \\
& \quad\left\langle\left|E_{r}\left(\lambda_{m}, \theta_{n}\right)\right|^{2}\right\rangle+\left\langle\left|E_{s}\left(\lambda_{m}, \theta_{n}\right)\right|^{2}\right\rangle \\
& \quad+2 \operatorname{Re}\left\langle E_{s}\left(\lambda_{m}, \theta_{n}\right) E_{r}^{*}\left(\lambda_{m}, \theta_{n}\right) \cos (\phi)\right\rangle
\end{aligned}
$$

where $\phi$ is the phase difference between the two fields, $(m, n)$ denotes a pixel on the CCD, and $<>$ denotes a temporal average. $I\left(\lambda_{m}, \theta_{n}\right)$ is uploaded to a PC using LabVIEW (Nat. Inst.) software and processed in 320 ms to produce a depth and angle resolved contour plot of scattered intensity [21].

\section{Angle-resolved low coherence interferometry (a/LCI) - Data analysis}

Measured a/LCI data from the systems described above consist of the angular distribution of scattered light, which contains information about the tissue structure, as a function of depth within the tissue. The light scattered from a particular depth is selectively detected using coherence gating. The effects of scattering by layers superficial to those of interest have been discussed previously [27] and are negligible for typical depths of 50-100 $\mu \mathrm{m}$ selected for analysis of epithelial tissues.

The diameter of the a/LCI probe beam, approximately 400-500 $\mu \mathrm{m}$ with all implemented systems, enables a single layer of a few hundred cells to be sampled at once, providing an average nuclear morphology measurement within the probe volume. The use of an average minimizes the effect of cell to cell variations in nuclear morphology such as irregular nuclear shape and variations in density arising from differing phases of the reproductive cycle.

Because a/LCI obtains the scattering distribution as a function of depth, the selection of a region of interest for analysis is an important consideration. In squamous epithelial tissues, the basal cell layer of the esophageal epithelium has been found to be a diagnostically useful ROI. The basal layer is identified by the topmost sharp peak in the density profile of the tissue, obtained by integrating the a/LCI data over angle. We note that a thickened keratin layer may be observed for some tissue samples which can cause the basal layer to be as deep as $200 \mu \mathrm{m}$. Although multiple scattering is more significant at these depths, previous studies have shown that the oscillations in the angular distribution used to determine size are still evident [27]. In our studies of tissues from the respiratory tract, the epithelium presents as a single cell layer atop the stroma. In this case, the topmost layer is chosen for analysis. In more complex tissue architectures, such as columnar epithelium, selection of an ROI may be a more difficult task. In our studies with human epithelial tissues from Barrett's esophagus (BE) patients [17], a summary measure of the average nuclear morphology in the superficial $150 \mu \mathrm{m}$ was used.

For the experimental studies, the a/LCI data from the selected ROI of each sample was processed and analyzed to determine the mean diameter of the cell nuclei, as previously described [30]. In brief, a/LCI data consist of an oscillatory component superposed on a slowly varying background. The slowly varying feature of the a/LCI data was modeled using a second-order polynomial and removed. The resulting oscillatory component was then compared to a database of theoretical predictions obtained using Mie theory from which the slowly varying features were similarly removed for analysis. The theoretical predictions were calculated for a Gaussian distribution of sizes characterized by a mean diameter $(d)$ and standard deviation $(\delta \mathrm{D})$. In addition, the refractive index of the nuclei relative to the cytoplasm was varied from $1.015-1.072$ in 36 discrete steps. The a/LCI technique is most sensitive to changes in mean nuclear diameter, the descriptor of nuclear morphology featured in our previous studies [2, $17,18,28,30,31]$; however, useful information may also be obtained from the refractive index measurements as will be shown below.

\section{Animal models and sources of tissues}

There were three sources of tissues used in the studies described here: two animal models and ex vivo human tissues. All of the experimental protocols were in accordance with NIH guidelines and approved by the Institutional Animal Care and Use Committees of Duke University. The human tissue studies were deemed exempt from IRB approval as they were ex vivo pathology specimens.

The first study used tissues drawn from the rat esophagus carcinogenesis model. Male F344 rats, 4-5 weeks of age, were obtained from Harlan Sprague Dawley (Indianapolis, IN). Carcinogen-treated animals were given subcutaneous injections of NMBA $(0.25 \mathrm{mg} / \mathrm{kg}$ body weight), obtained from Ash Stevens, Inc. (Detroit, MI), in the intrascapular region three times a week for 5 weeks with concentrations adjusted weekly based up- 
on average body weight. The solvent for NMBA was $20 \%$ DMSO: $\mathrm{H}_{2} \mathrm{O}$ and the injection volume was $0.2 \mathrm{ml}$. Control groups received a similar injection regimen of 20\% DMSO: $\mathrm{H}_{2} \mathrm{O}$. At 8, 12 and 20 weeks after the initial injection of NMBA, NMBA-treated and control rats were harvested for optical spectroscopic analysis. The rats were euthanized by $\mathrm{CO}_{2}$ asphyxiation and subjected to gross necropsy. The entire esophagus was excised and opened longitudinally for immediate optical spectroscopic analysis. Following spectroscopic analysis, the esophagus was fixed in $10 \%$ neutral buffered formalin.

The second study described here used tissues from the Syrian golden hamster trachea carcinogenesis model. Male Syrian golden hamsters 6-7 weeks of age were obtained from Charles River Laboratories (Wilmington, MA). Carcinogen treated animals were administered MNU at a concentration of $0.5 \%$ once/week for ten weeks by intratracheal instillation. Ten weeks after completion of this dosing regimen, animals were euthanized by $\mathrm{CO}_{2}$ asphyxiation. Tracheas were excised and opened longitudinally for immediate spectroscopic analysis. After spectroscopic analysis, the tracheal samples were fixed in $10 \%$ neutral buffered formalin.

The ex vivo human tissues used for the studies here were obtained from three BE patients who underwent esophago-gastrectomies. The tissue was opened longitudinally, after excision, and data were taken within two hours. Scanned areas were bracketed with India ink to allow for comparison of the a/LCI data to subsequent pathological classification by a trained pathologist.

\section{Statistical analysis}

For each histological classification, the mean and standard deviation from the mean of the a/LCI nuclear size measurements across samples was determined. Additionally, the mean and standard deviation from the mean of the refractive index of the nucleus relative to the cytoplasm across samples was established. To assess the diagnostic capacity of the a/LCI method, doubled-sided t-tests were performed with the online software offered by Simple Interactive Statistical Analysis (http://home.clara.net/sisa/t-thlp.htm) to compare the a/LCI morphological descriptors. The nuclear size and refractive index of the nucleus relative to the cytoplasm were compared between the classifications; the statistical difference was quantified by the p-value obtained from the t-test (reported below).

\section{Results}

Each of the a/LCI spectra is processed and analyzed to determine the nuclear morphology of the associated sample. In previous studies [2,17,28,31], the chief morphological descriptor used for classifying tissues was the mean size of the cell nuclei. Here we also report the relative refractive index as a means to assess its diagnostic capacity as a biomarker.

The first a/LCI study of rat esophagus [28] reported the a/LCI measured nuclear morphology and its correlation with histology. In this study, the data (Fig. 3) showed an increase in mean nuclear size with neoplastic progression. The average nuclear size in normal tissues was found to be $9.55 \pm 0.23 \mu \mathrm{m}$, increases to 10.5 $\pm 0.56 \mu \mathrm{m}$ for LGD and to $14.4 \pm 0.21 \mu \mathrm{m}$ for HGD. A statistically significant difference was found between the normal and LGD mean sizes $(p<0.001)$, and between normal and abnormal (LGD + HGD) mean sizes $(p<0.005)$. A decision threshold was established using logistic regression, which yielded $80 \%$ sensitivity and $100 \%$ specificity in distinguishing normal and dysplastic tissues. The original study did not report the refractive index of the nucleus relative to the cytoplasm obtained from the a/LCI data. The relative refractive index of the nuclei in normal tissues was found to be $1.052 \pm 0.0051$ and that of the dysplastic tissues was found to be $1.051 \pm 0.0082$. This is not a statistically significant difference ( $p=0.74)$. However, visual inspection of the data in Fig. 2 reveals an obvious outlier. Upon removal of this point from analysis, the average for the dysplastic tissues drops to $1.049 \pm 0.0031$. Although this result is still not statistically significant, the p-value drops dramatically to $p=0.07$.

The second a/LCI study of rat esophagus [31] was a prospective study which used the decision line from the first study to classify tissues. This study consisted of two cohorts of animals examined at several time points. The data were originally presented for the mean nuclear size as a function of neoplastic progression and at multiple time points. Here we combine the data across time points as a single diagnostic metric. For the first cohort of animals in the second study, the mean nuclear diameter was found to be $9.09 \pm$ $1.06 \mu \mathrm{m}$ for the normal tissues and $11.86 \pm 1.16 \mu \mathrm{m}$ for the dysplastic tissues a highly statistically significant difference $(p<0.0001)$. The relative refractive index of the nuclei in these tissues was found to be $1.047 \pm$ 0.0051 for the normal tissues and $1.041 \pm 0.0038$ for the dysplastic tissues. This was also a highly statistically significant difference $(p<0.001)$. In the second cohort 
Rat Esophagus Study I

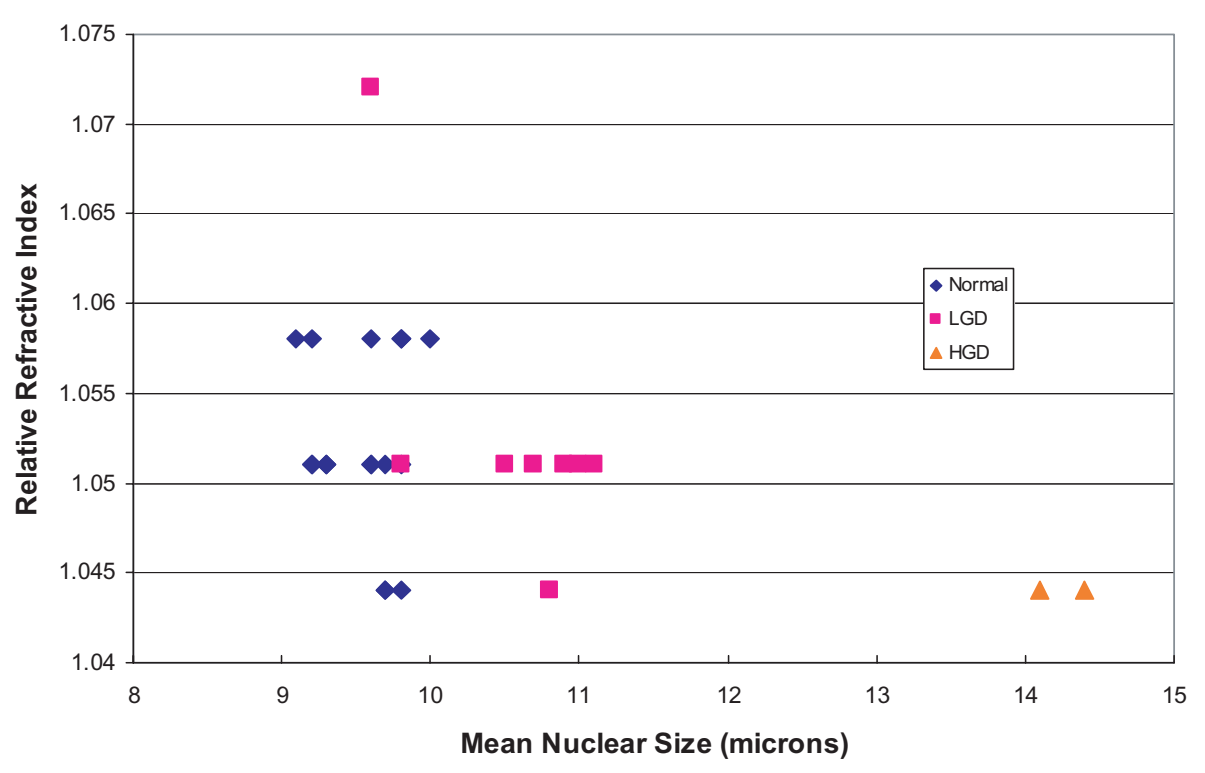

Fig. 3. Nuclear morphology of epithelial tissues from the rat esophagus.

of animals in this study, the mean nuclear diameter was found to be $8.80 \pm 0.51 \mathrm{~mm}$ for normal tissues and $12.07 \pm 1.73 \mathrm{~mm}$ for dysplastic tissues, a highly statistically significant difference $(p<0.0001)$. The relative refractive index of the nuclei in these tissues was found to be $1.057 \pm 0.0068$ for the normal tissues and $1.051 \pm 0.0094$ for the dysplastic tissues. This difference was not found to be statistically significant but is comparable to the significance in the first study $(p=0.077)$.

The a/LCI study of neoplastic progression in hamster trachea [2] was a pilot study and only contained a limited number of samples. This study reported both the mean nuclear diameter and relative refractive index for normal and dysplastic tissues, shown graphically in Fig. 4. The mean nuclear diameter was found to be $7.00 \pm 1.09 \mu \mathrm{m}$ for the normal tissues and increased to $11.19 \pm 3.2 \mu \mathrm{m}$ for the dysplastic tissues, a statistically significant difference $(p=0.03)$. The relative refractive index of the cell nuclei was found to be 1.057 \pm 0.0064 for the normal tissues and $1.048 \pm 0.012$ for the dysplastic tissues. This difference was not found to be statistically significant but was consistent with the significance of the studies above $(p=0.06)$.

The results of the a/LCI study of human esophageal tissues [17] are shown in Fig. 5. The mean nuclear diameter in the superficial $150 \mu \mathrm{m}$ of tissue was found to be $10.05 \pm 0.72 \mu \mathrm{m}$ for normal columnar tissue and rose to $12.86 \pm 1.44 \mu \mathrm{m}$ for dysplastic Barrett's esoph- agus tissues, a highly statistically significant difference $(p<0.0001)$. The relative refractive index of the nuclei was found to be $1.046 \pm 0.0007$ for the normal columnar tissues and decreased to $1.037 \pm 0.0047$ for the dysplastic Barrett's esophagus tissues. This difference was also found to be highly statistically significant $(p<0.0001)$.

\section{Discussion}

The use of nuclear morphology is a potentially powerful method for diagnosing dysplastic tissues. Here we have reviewed the use of a/LCI for obtaining nuclear morphology measurements from epithelial tissues. The a/LCI method offers the advantage that no tissue sectioning, fixation or staining agents are required. This advantage has obvious benefits for in vivo application to detecting dysplasia in human tissues. However, the a/LCI method has also been applied to assessing neoplastic changes in animal tissues, notably the rat esophagus carcinogenesis model and more recently in the hamster trachea carcinogenesis model. Here the a/LCI method offers several benefits over traditional analysis as well. First, the ability to obtain nuclear morphology data without tissue processing enables additional immunohistochemistry studies which may be prevented upon tissue fixation. Further, the a/LCI method has been shown capable of detecting neoplastic 


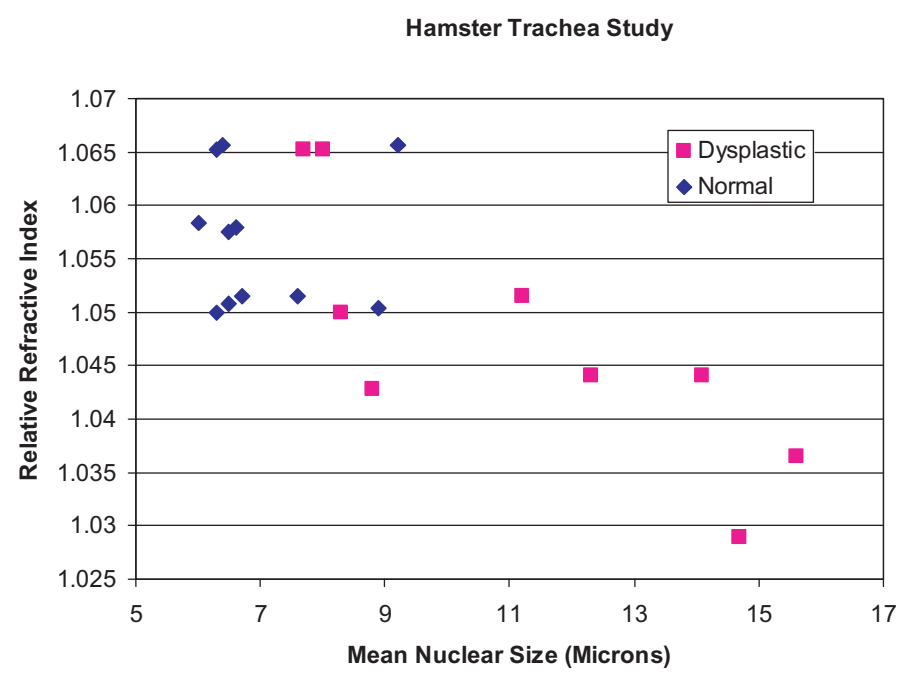

Fig. 4. Nuclear morphology of epithelial tissues from the hamster trachea.

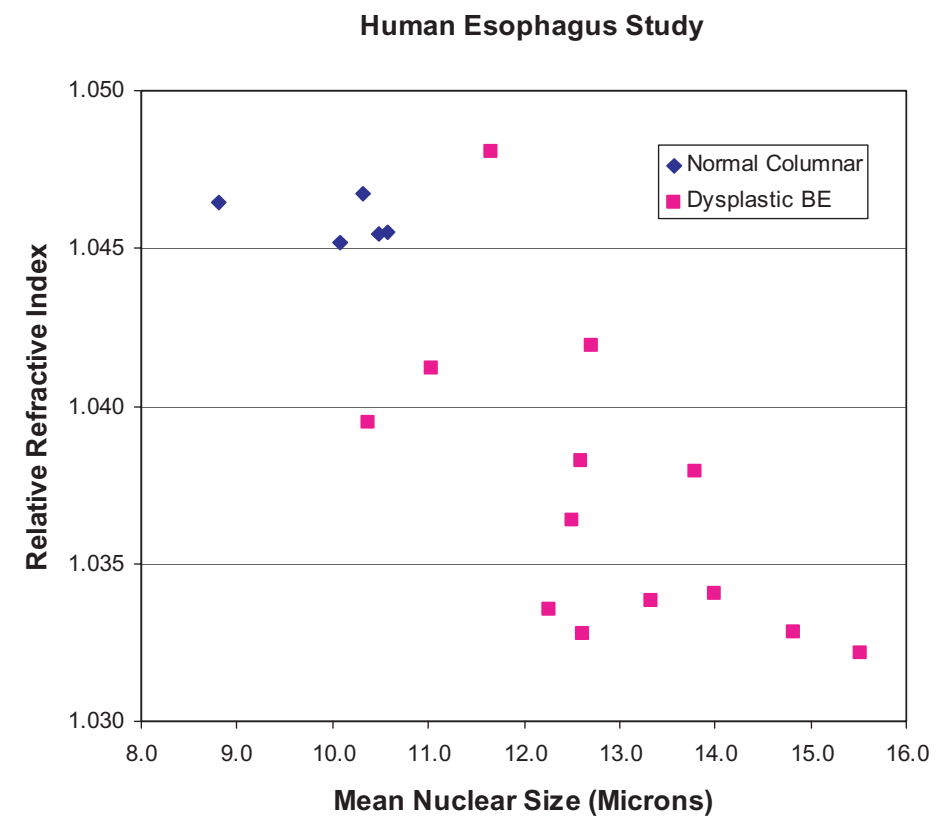

Fig. 5. Nuclear morphology of human epithelial tissues from Barrett's esophagus patients.

changes earlier than other bioassay methods, such as tumor metrology measurements [31]. Finally, because a/LCI can detect unusual cell states such as apoptosis $[28,31]$, the method can be applied to assessing the efficacy of candidate chemopreventive agents in animal models.

The review presented above recalled the diagnostic power of the a/LCI technique when using the mean nuclear diameter as a biomarker. In our previous studies $[2,17,28,31]$, the mean nuclear diameter has been used to obtain high sensitivity and specificity, typically between $80-96 \%$. This diagnostic accuracy has spurred the development of a/LCI as a clinical diagnostic method [19,21]. Although these studies have demonstrated high diagnostic accuracy, it became evident that when assessing tissue health in complicated architectures such as those found in Barrett's esophagus tissues that additional information could improve the diagnostic capacity. In our study of Barrett's esophagus tissue reviewed above, it was shown that diagnos- 
tic power was improved by incorporating a second descriptor of nuclear morphology, specifically the relative refractive index of the cell nuclei relative to the cytoplasm, a parameter related to the density of chromatin within the cell nucleus.

Multiple morphological descriptors have been used previously in a/LCI studies. In the a/LCI studies of the rat esophagus reviewed above, a second morphological descriptor was reported in the original publications [28, 31]. Specifically, the fractal dimension of the structures within the cell nucleus, a parameter which has been given the physical interpretation of the nucleus granularity, was reported. This parameter was found to be statistically significant in some of the subgroups within the studies but in general, did not add to the diagnostic capacity of the measurement. Although the use of fractal dimension has produced some interest in recent years [10,23], the a/LCI method has not obtained sufficiently precise measurements of this parameter for warranting its inclusion in diagnostic analysis. This lack of precision is most likely due to the fact that with $\mathrm{a} / \mathrm{LCI}$ the fractal dimension is obtained from the residual of the signal and measurement of this parameter can be greatly influenced by noise.

As an alternative to the use of fractal dimension, our more recent studies $[2,17]$ have incorporated the relative refractive index of the cell nuclei as a morphological descriptor, usually interpreted as the density of the cell nuclei. In contrast to the use of fractal dimension, the refractive index parameter was found to produce fairly consistent statistical significance and was found to impact the diagnostic power of the a/LCI method. Although only a couple of studies reviewed above showed that the difference in relative refractive index of the cell nuclei in normal and dysplastic tissue was statistically significant using a strict definition of a p-value less than 0.05 , it was found that all cases had a similar significance, yielding p-values between 0.06 and 0.08 . Based on this limited statistical significance, it is unlikely that relative refractive index will offer sufficient diagnostic power to be used as a sole criterion of tissue health. However, when coupled with measurements of mean nuclear diameter, the use of relative refractive index can serve to improve diagnostic power. Upon examining the data presented in Figs 4 and 5 , we see that diagnostic power can be improved by using both parameters in establishing a decision line. In both of these figures, diagonal lines will improve separation between normal and dysplastic tissues to a greater degree than a simple horizontal or vertical line.

Analysis of the relative refractive index data presented above reveals a trend that shows a decrease in this de- scriptor with neoplastic progression. It is interesting to note that although several other studies have employed light scattering measurements of nuclear morphology as a means to diagnose dysplasia $[6,7,16]$, this trend had not been previously identified. The demonstration of the utility of relative refractive index may serve to warrant its inclusion in further light scattering studies of nuclear morphology.

\section{Conclusion}

We have reviewed the use of a/LCI nuclear morphology measurements for detecting dysplastic tissues. The a/LCI method has been applied successfully to monitoring neoplastic progression in studies of animal tissues, such as the rat esophagus carcinogenesis model. Here we have emphasized the use of a new morphological descriptor, the refractive index of cell nuclei relative to their surroundings. When coupled with the use of mean nuclear diameter, the two parameters can offer significant diagnostic power for detecting dysplastic tissues. Based on the high sensitivity and specificity obtained in animal studies, we have begun to translate a/LCI for clinical applications such as the detection of dysplasia in Barrett's esophagus patients. Preliminary results reviewed here suggest that a/LCI may offer a powerful new method for detecting dysplastic tissues in the clinic.

\section{Acknowledgements}

This work was supported by grants from Hamamatsu Corporation, the National Institutes of Health through the National Center for Research Resources (P41-RR02594) and the National Cancer Institute (NCI R21-CA109907, R33-109907, CN15011-72, N01-CN43308) and the National Science Foundation (BES 0348204, CHE-0111370)

\section{References}

[1] B.C. Casto, L.A. Kresty, C.L. Kraly, D.K. Pearl, T.J. Knobloch, H.A. Schut, G.D. Stoner, S.R. Mallery and C.M. Weghorst, Chemoprevention of oral cancer by black raspberries, Anticancer Research 22(6C) (2002), 4005-4015.

[2] K.J. Chalut, L.A. Kresty, J.W. Pyhtila, R. Nines, M. Baird, V.E. Steele and A. Wax, In situ assessment of intraepithelial neoplasia in hamster trachea epithelium using angle-resolved low coherence interferometry, Cancer Epidemiol Biomarkers Prev 16 (2007), 223-227. 
[3] Y. Chen, G. Zheng, Z.H. Zhang, D. Blessington, M. Zhang, H. Li, Q. Liu, L. Zhou, X. Intes, S. Achilefu and B. Chance, Metabolism-enhanced tumor localization by fluorescence imaging: in vivo animal studies, Optics Letters $\mathbf{2 8}(21)$ (2003), 2070-2072.

[4] G. Choy, S. O'Connor, F.E. Diehn, N. Costouros, H.R. Alexander, P. Choyke and S.K. Libutti, Comparison of noninvasive fluorescent and bioluminescent small animal optical imaging, Biotechniques 35(5) (2003), 1022-1030.

[5] C. Dwivedi, L.A. Muller, D.E. Goetz-Parten, K. Kasperson and V.V. Mistry, Chemopreventive effects of dietary mustard oil on colon tumor development, Cancer Letters 196(1) (2003), 29-34

[6] I. Georgakoudi, B.C. Jacobson, J. Van Dam, V. Backman, M.B. Wallace, M.G. Muller, Q. Zhang, K. Badizadegan, D. Sun, G.A. Thomas, L.T. Perelman and M.S. Feld, Fluorescence, reflectance, and light-scattering spectroscopy for evaluating dysplasia in patients with Barrett's esophagus, Gastroenterology 120(7) (2001), 1620-1629.

[7] I. Georgakoudi, E.E. Sheets, M.G. Muller, V. Backman, C.P. Crum, K. Badizadegan, R.R. Dasari and M.S. Feld, Trimodal spectroscopy for the detection and characterization of cervical precancers in vivo, American Journal of Obstetrics and Gynecology 186(3) (2002), 374-382.

[8] M. Hiroyasu, M. Ozeki, A. Miyagawa-Hayashino, Y. Fujiwara, H. Hiai and S. Toyokuni, Novel surrogate end-point biomarker to evaluate agents for use in the chemoprevention of reactive oxygen species-associated cancer, Redox Report 7(5) (2002), 335-338.

[9] D. Huang, E.A. Swanson, C.P. Lin, J.S. Schuman, W.G. Stinson, W. Chang, M.R. Hee, T. Flotte, K. Gregory, C.A. Puliafito, and J.G. Fujimoto, Optical Coherence Tomography, Science 254(5035) (1991), 1178-1181.

[10] M. Hunter, V. Backman, G. Popescu, M. Kalashnikov, C.W. Boone, A. Wax, V. Gopal, K. Badizadegan, G.D. Stoner and M.S. Feld, Tissue self-affinity and polarized light scattering in the Born approximation: A new model for precancer detection, Physical Review Letters 97(13) (2006).

[11] J.A. Izatt, M.D. Kulkarni, H.W. Wang, K. Kobayashi and M.V. Sivak, Optical coherence tomography and microscopy in gastrointestinal tissues, Ieee Journal of Selected Topics in Quantum Electronics 2(4) (1996), 1017-1028.

[12] A. Izzotti, R.M. Balansky, F. D’Agostini, C. Bennicelli, S.R. Myers, C.J. Grubbs, R.A. Lubet, G.J. Kelloff and S. De Flora, Modulation of biomarkers by chemopreventive agents in smoke-exposed rats, Cancer Research 61(6) (2001), 24722479.

[13] L.M. Kalikin, A. Schneider, M.A. Thakur, Y. Fridman, L.B. Griffin, R.L. Dunn, T.J. Rosol, R.B. Shah, A. Rehemtulla, L.K. McCauley and K.J. Pienta, In vivo visualization of metastatic prostate cancer and quantitation of disease progression in immunocompromised mice, Cancer Biology \& Therapy 2(6) (2003), 656-660.

[14] T.W. Kensler, S.J. Gange, P.A. Egner, P.M. Dolan, A. Munoz, J.D. Groopman, A.E. Rogers and B.D. Roebuck, Predictive value of molecular dosimetry: Individual versus group effects of oltipraz on aflatoxin-albumin adducts and risk of liver cancer, Cancer Epidemiology Biomarkers \& Prevention 6(8) (1997), 603-610.

[15] B.W. Liston, R. Nines, P.S. Carlton, A. Gupta, R. Aziz, W. Frankel and G.D. Stoner, Perillyl alcohol as a chemopreventive agent in N-nitrosomethylbenzylamine-induced rat esophageal tumorigenesis, Cancer Research 63(10) (2003), 2399-2403.
[16] L.T. Perelman, V. Backman, M. Wallace, G. Zonios, R. Manoharan, A. Nusrat, S. Shields, M. Seiler, C. Lima, T. Hamano, I. Itzkan, J. Van Dam, J.M. Crawford, and M.S. Feld, Observation of periodic fine structure in reflectance from biological tissue: A new technique for measuring nuclear size distribution, Physical Review Letters 80(3) (1998), 627-630.

[17] J.W. Pyhtila, K.J.Chalut, J.D. Boyer, J. Keener, T.A. D'Amico, M.A Gottfried, F. Gress and A. Wax, In situ detection of nuclear atypia in Barrett's esophagus using angle-resolved low coherence interferometry, Gastrointestinal Endoscopy 65 (2007), 487-491.

[18] J.W. Pyhtila, M.H. Simnick, A.J. Chilkoti and A. Wa, Analysis of long range correlations due to coherent light scattering from in-vitro cell arrays using angle-resolved low coherence interferometry, Journal of Biomedical Optics 11 (2006), 034022.

[19] J.W. Pyhtila and A. Wax, Polarization effects on scatterer sizing accuracy analyzed with frequency-domain angle-resolved low coherence interferometry, in press, 2007.

[20] J.W. Pyhtila and A. Wax, Rapid, depth-resolved light scattering measurements using Fourier domain, angle-resolved low coherence interferometry, Optics Express 12(25) (2004), 6178-6183.

[21] J.W. Pyhtila, J.D. Boyer, K.J. Chalut and A. Wax, Fourierdomain angle-resolved low coherence interferometry through an endoscopic fiber bundle for light-scattering spectroscopy, Optics Letters 31(6) (2006), 772-774.

[22] J.W. Pyhtila, R.N. Graf and A. Wax, Determining nuclear morphology using an improved angle-resolved low coherence interferometry system, Optics Express 11(25) (2003), 34733484.

[23] H.K. Roy, Y. Liu, R.K. Wali, Y.L. Kim, A.K. Kromine, M.J. Goldberg and V. Backman, Four-dimensional elastic lightscattering fingerprints as preneoplastic markers in the rat model of colon carcinogenesis, Gastroenterology 126(4) (2004), 1071-1081.

[24] W.A. Smith and R.C. Gupta, Determining efficacy of cancer chemopreventive agents using a cell-free system concomitant with DNA adduction, Mutation Research-Fundamental and Molecular Mechanisms of Mutagenesis 425(1) (1999), 143152.

[25] J. Song, K.J. Sohn, A. Medline, C. Ash, S. Gallinger and Y.I. Kim, Chemopreventive effects of dietary folate on intestinal polyps in Apc \pm Msh2 - / - mice, Cancer Research 60(12) (2000), 3191-3199.

[26] A. Wax and J.E. Thomas, Optical heterodyne imaging and Wigner phase space distributions, Optics Letters 21(18) (1996), 1427-1429.

[27] A. Wax, C. Yang, V. Backman, M. Kalashnikov, R.R. Dasari and M.S. Feld, Determination of particle size using the angular distribution of backscattered light as measured with lowcoherence interferometry, Journal of the Optical Society of America a-Optics Image Science and Vision 19 (2002), 737744.

[28] A. Wax, C.H. Yang, M.G. Muller, R. Nines, C.W. Boone, V.E. Steele, G.D. Stoner, R.R. Dasari and M.S. Feld, In situ detection of neoplastic transformation and chemopreventive effects in rat esophagus epithelium using angle-resolved lowcoherence interferometry, Cancer Research 63(13) (2003), 3556-3559.

[29] A. Wax, C.H. Yang, R.R. Dasari and M.S. Feld, Measurement of angular distributions by use of low-coherence interferometry for light-scattering spectroscopy, Optics Letters 26(6) (2001), 322-324. 
[30] A. Wax, C.H. Yang, V. Backman, K. Badizadegan, C.W. Boone, R.R. Dasari, and M.S. Feld, Cellular organization and substructure measured using angle- resolved low-coherence interferometry, Biophysical Journal 82(4) (2002), 2256-2264.

[31] A. Wax, J.W. Pyhtila, R.N. Graf, R. Nines, C.W. Boone, R.R.
Dasari, M.S. Feld, V.E. Steele and G.D. Stoner, Prospective grading of neoplastic change in rat esophagus epithelium using angle-resolved low-coherence interferometry, Journal of Biomedical Optics 10(5) (2005), 051604. 


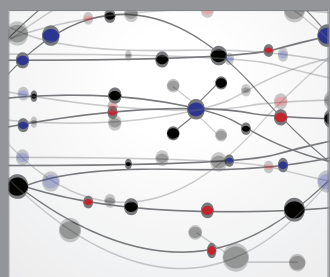

The Scientific World Journal
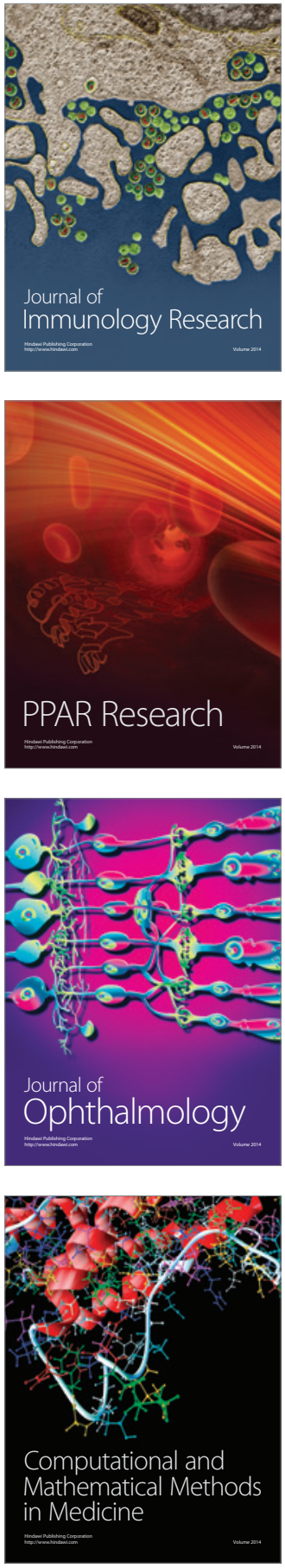

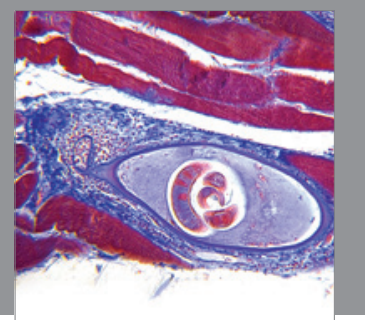

Gastroenterology

Research and Practice
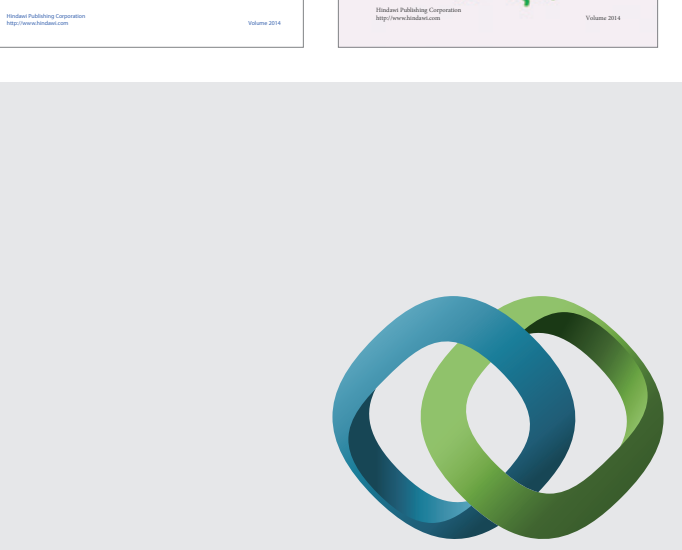

\section{Hindawi}

Submit your manuscripts at

http://www.hindawi.com
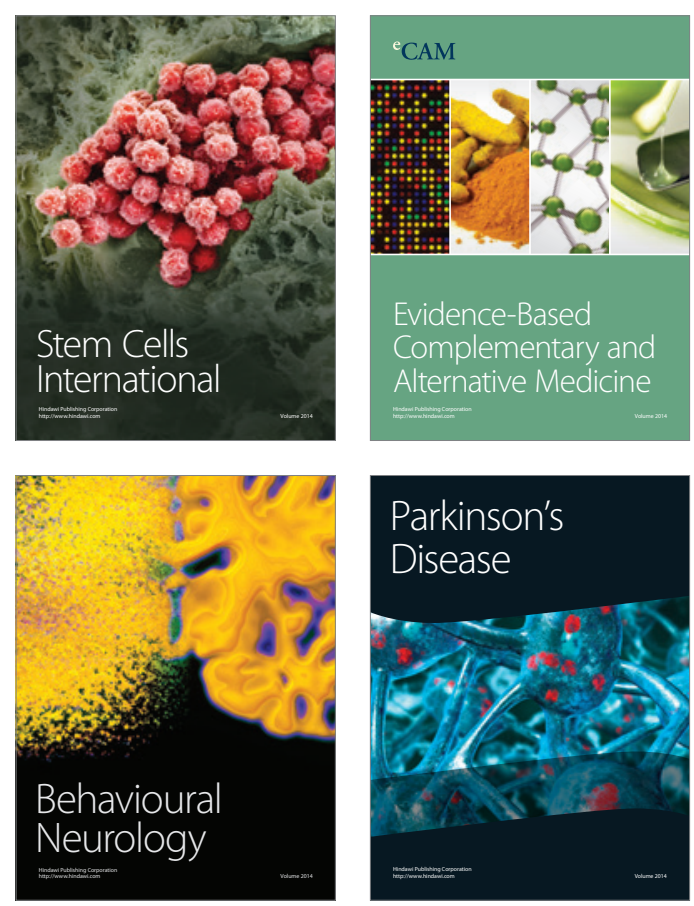

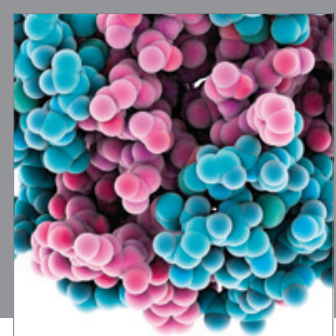

Journal of
Diabetes Research

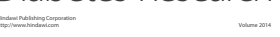

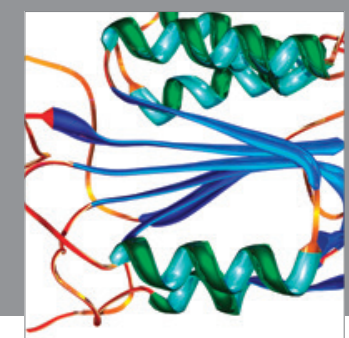

Disease Markers
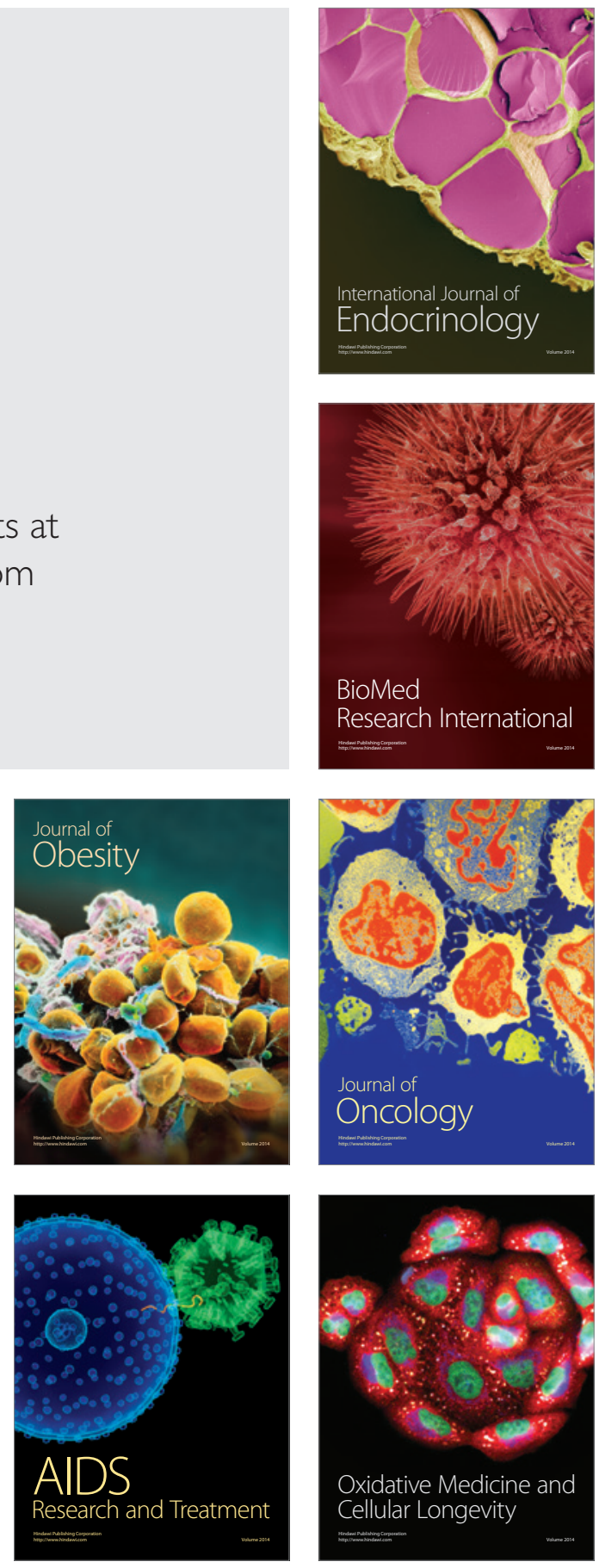